\title{
MicroRNA Regulation of Brown Adipogenesis and Thermogenic Energy Expenditure
}

\author{
Farnaz Shamsi', Hongbin Zhang ${ }^{2}$ and Yu-Hua Tseng ${ }^{1,3 *}$ \\ ${ }^{1}$ Section on Integrative Physiology and Metabolism, Joslin Diabetes Center, Harvard Medical School, Boston, MA, \\ United States, ${ }^{2}$ Department of Biomedical Sciences, University of Copenhagen, Copenhagen, Denmark, ${ }^{3}$ Harvard Stem \\ Cell Institute, Harvard University, Cambridge, MA, United States
}

OPEN ACCESS

Edited by:

Lei Sun,

National University of Singapore, Singapore

Reviewed by:

Feng $\mathrm{Xu}$,

Singapore Institute for

Clinical Sciences

(A*STAR), Singapore

Li Qiang,

Columbia University,

United States

${ }^{*}$ Correspondence:

Yu-Hua Tseng

yu-hua.tseng@joslin.harvard.edu

Specialty section:

This article was submitted to Endocrinology of Aging, a section of the journal

Frontiers in Endocrinology

Received: 25 June 2017 Accepted: 07 August 2017

Published: 23 August 2017

Citation:

Shamsi F, Zhang $H$ and Tseng $Y-H$

(2017) MicroRNA Regulation

of Brown Adipogenesis

and Thermogenic Energy

Expenditure.

Front. Endocrinol. 8:205.

doi: 10.3389/fendo.2017.00205
Obesity, diabetes, and associated metabolic diseases have become global epidemics. Obesity results from excess accumulation of white fat, while brown and its related beige fat function to dissipate energy as heat, thus counteracting obesity and its related metabolic disorders. Understanding the regulatory mechanisms for both white and brown adipogenesis provides new insights for prevention and treatment of these metabolic diseases. In addition to traditional gene transcription and translation, microRNA (miRNA) represents a new layer of regulatory mechanism in many biological processes and has attracted a great deal of research interests in exploring their roles in physiological and pathophysiological conditions. This review focuses on the recent advances of regulating brown adipogenesis and energy metabolism by miRNAs, aiming to delineate the regulatory principles of miRNAs on this unique aspect of energy homeostasis.

Keywords: microRNA, adipose tissue, brown, non-coding RNAs, uncoupling protein 1, adipogenesis, gene expression regulation

\section{INTRODUCTION}

Obesity has become a global epidemic and major contributor to metabolic syndrome and disorders such as type 2 diabetes, cardiovascular disease, Alzheimer's disease, and many cancers. There are two types of adipose tissues in human body: white adipose tissue (WAT) and brown adipose tissue (BAT). WAT is specialized to store excess energy in the form of triglycerides and plays a pivotal role in the regulation of energy homeostasis. In addition, WAT is the biggest endocrine organ in the body and secretes several adipocyte-derived hormones, such as adiponectin $(1)$, leptin $(1,2)$, resistin (3), and others, which regulate insulin sensitivity, appetite, glucose, and lipid metabolism. $\mathrm{BAT}$, on the other hand, is the key site for non-shivering thermogenesis and has a unique capacity to dissipate excess fuel energy as heat. BAT has high mitochondrial density and expresses uncoupling protein 1 (UCP1). UCP1 is a proton channel, localized to the inner mitochondrial membrane that allows protons in the mitochondrial intermembrane space to reenter the mitochondrial matrix without generating ATP. BAT-mediated thermogenesis plays a crucial role in thermostatic regulation, particularly when facing environmental changes such as cold and diet. In addition, BAT possesses an enormous capacity for glucose uptake and plays an important function in both lipid and glucose metabolism (4). Functional BAT has long thought to exist only in newborns but was recently rediscovered in adult humans (5-7). In addition to classical BAT, cold exposure, exercise training and other types of stimulation induce the formation of a type of brown-like adipocytes (known as beige or brite adipocytes) within WAT (a process called "browning" or "beiging" of WAT) $(8,9)$, which expresses UCP1 and has thermogenic capacity comparable to BAT. Because 
BAT counteracts energy storage in WAT by promoting energy expenditure, enhancing the development and activity of BAT and beige fat has become an attractive potential strategy to prevent and treat human obesity.

\section{DEVELOPMENTAL ORIGIN OF BROWN AND BEIGE FAT}

Adipocyte differentiation is a coordinated process regulated by a series of transcriptional cascades consisting of both positive and negative regulators. In addition to general adipogenic regulators, brown adipocytes require specific brown fat lineage commitment factors. All these regulators are expressed in a temporal manner along with brown adipocyte differentiation process. Classical interscapular BAT is developed from precursors in the embryonic mesoderm that give rise to both BAT and skeletal muscle cells. These multipotent precursor cells express transcription factors such as paired box 7 (10), engrailed-1 (11), and myogenic factor 5 (MYF5) (12). Lineage tracing experiments have shown that the majority of beige adipocytes in the subcutaneous white fat depot (scWAT) come from a developmentally distinct lineage that lack MYF5 expression (12).

Transition of adipocyte precursors (preadipocytes) to mature adipocytes is orchestrated by a cascade of transcription regulators such as peroxisome proliferator-activated receptor gamma (PPAR $\gamma)$ and members of the CCAAT/enhancer-binding protein family (C/EBPs) (13). In addition to general regulators of adipogenesis essential for both brown and white adipocyte development, the expression of thermogenic gene program in brown/ beige adipocytes is regulated by additional transcriptional regulators such as peroxisome proliferative activated receptor gamma coactivator 1 alpha (14), PR domain containing 16 (PRDM16) (15), forkhead box C2 (16), and others.

\section{MicroRNAs (miRNAs) ROLES IN FINE- TUNING OF GENE EXPRESSION}

MicroRNAs are a class of short non-coding RNAs consisting of 22 nucleotides and represent a new layer of fundamental regulatory mechanism for transcription and translation (17). miRNAs are key regulators of diverse biological processes, such as proliferation and differentiation and are also involved in the pathophysiology of many diseases (18). In general, miRNAs function as negative regulators of gene expression. They are usually transcribed by RNA polymerase II as primary miRNAs (pri-miRNAs), and then processed by Drosha RNAse III endonuclease and microprocessor complex subunit DGCR8 to generate precursor miRNAs (pre-miRNAs) that are about 60-70 nucleotides long. Following Exportin-5-mediated transport to the cytoplasm, pre-miRNAs are cleaved by a Dicer complex to generate the miRNA/miRNA* double strand. Eventually, mature miRNAs will be loaded into the miRNA-induced silencing complex where they target and bind to the 3' UTR of specific genes, leading either to mRNA degradation or translational repression (19).

Over the past few years, the role of miRNAs in the regulation of different biological processes has become evident. Numerous studies have pointed to the significance of miRNAs in the regulation of adipose tissue development and function. In this review, we discuss the multiple roles of miRNAs in brown and beige fat biology (Figure 1). In addition, we discuss the newly discovered function of secreted miRNAs as metabolic

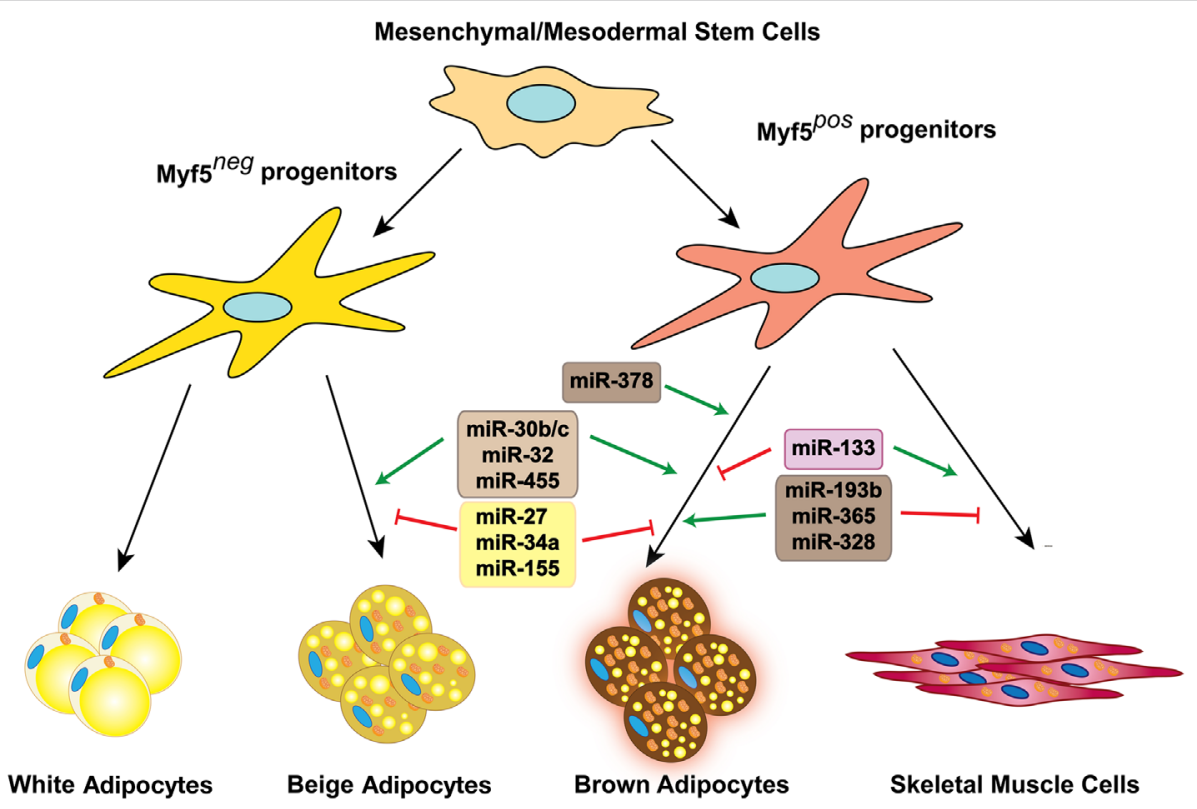

FIGURE 1 | miRNAs involved in brown and beige fat development and function. Several miRNAs positively or negatively regulate brown fat lineage determination and differentiation, as well as beige fat development. 
messengers, enabling communication between different tissues and organs in the body.

Normal miRNA processing has been shown to be essential for the maintenance of brown and WAT function. Aging results in a global downregulation of mature miRNAs in WAT, which is attributed to decreased expression of miRNA processing enzymes, mainly Dicer. This massive loss of miRNAs could be largely prevented by caloric restriction, which is known to expand lifespan in mice and many other species by up to $60 \%$ (20). Mice lacking Dicer expression in adipose tissue displayed an abnormal fat accumulation in the interscapular fat depot and decreased mass of the subcutaneous and intra-abdominal fat depots, resembling HIV-associated lipodystrophy phenotype. This was accompanied by whitening of BAT and resulted in severe insulin resistance and dyslipidemia $(21,22)$. Consistently, partial reduction of Dicer expression in BAT, by deleting only one copy of the gene, impaired BAT thermogenic function and exacerbated the effects of high fat diet-induced obesity on glucose metabolism (23). These observations clearly showed that Dicer function is essential for development of WAT and maintaining BAT identity and function. However, recent studies have provided strong evidence for miRNA-independent roles of Dicer $(24,25)$. The earliest hints supporting this came from studies showing different phenotypes of Dicer- and Droshadeficient cells. Therefore, to precisely elucidate the significance of miRNA biogenesis in adipose tissue development, additional animal models lacking other key miRNA processing enzymes would be crucial.

\section{miRNAs AS ACTIVATORS OF BROWN ADIPOGENESIS}

\section{miR-193b-365 Cluster}

miR-193b and miR-365 are BAT-enriched miRNAs conserved in both humans and mice (26). In mice, they are located within a 5 -kb distance on chromosome 16 and are transcribed as a bicistronic pri-miRNA (27). Chromatin immunoprecipitation experiments showed the binding of PPAR $\alpha$ to the PPAR $\alpha /$ RXR binding elements on the promoter region of this cluster. Blocking miR-193a/b and miR-365 inhibited brown adipocyte differentiation in vitro and resulted in a marked reduction in expression of key adipogenic genes including adiponectin, Cebpo, Fabp4, and Ppary, which is accompanied by a greater decrease in thermogenic genes such as Ucp1, Ppar $\alpha$, Ppargc1 $\alpha$, Dio2, Prdm16, and Cidea. These data suggest that miR-193b and miR-365 function is required for general adipogenesis pathways in addition to playing a role in development of brown adipocytes. miR-193b promotes induction of adipogenic versus myogenic fate, possibly by direct targeting and suppression of an adipogenesis inhibitor, Runx1t1, and two pro-myogenic genes, Cdon and Igfbp5. The physiological significance of these observations was later challenged by in vivo studies demonstrating the normal development and function of BAT in miR-193b KO mice. The discrepancy between the in vitro and in vivo findings might be partially explained by the compensatory downregulation of miR-133a, which acts as an inhibitor of brown adipogenesis (28) in BAT of miR-193b KO mice.

\section{miR-328}

To search for the individual miRNAs that regulate aging and obesity-associated decline in BAT function, Oliverio et al. integrated the expression of miRNAs in a mouse model of premature aging, long-lived Ames dwarf mutants, and diet-induced obese (DIO) mice. They identified miR-328 as a possible regulator of BAT function as its expression was induced in the BAT of the Ames dwarf mice and was decreased in the BAT of aging and DIO models (23). Loss of miR-328 resulted in the downregulation of BAT-selective genes such as Ucp1, Prdm16, Ppargc1 $\alpha$, Cidea, and Cebpo. Interestingly, the seed sequence of miR-328 is similar to that of miR-193b, another BAT-enriched miRNA described above, suggesting that they might share their target genes. Similar to miR-193b, miR-328 promotes brown adipocytes' differentiation, possibly by suppressing the expression of muscle lineage regulators. Mechanistically, miR-193b and miR328 target Bacel (beta-site amyloid precursor protein-cleaving enzyme 1), a muscle enriched gene that promotes myogenesis and inhibit brown fat commitment. Consistent with its function as a negative regulator of BAT differentiation and function, Bace 1 expression was induced in BAT of DIO mice and reduced by cold exposure. Moreover, inhibition of Bace1 enzymatic activity enhanced the expression of brown adipocyte markers in BAT and subcutaneous WAT.

\section{$\operatorname{miR}-378$}

miR-378 genomic locus is positioned in the sense strand of the first intron of Ppargc1 $\beta$, which is highly expressed in brown fat and is one of the key transcriptional regulators of mitochondrial biogenesis. Ectopic overexpression of miR-378 under the control of the aP2 promoter leads to expansion of BAT in mice, accompanied by a reduced mass of WAT depots. The BAT expansion is mainly the result of the enhanced differentiation of brown preadipocytes, and not an increase in cell size. In this model, BAT expansion prevented genetic and diet-induced obesity. Phosphodiesterase Pde1b is shown to be a direct target of miR-378 in BAT. miR-378-mediated downregulation of Pde1b enhances brown adipocyte differentiation by regulating cAMP turnover in BAT (29). miRNA profiling of abdominal subcutaneous adipose tissue from gastrointestinal cancer patients with or without cachexia identified upregulation of miR-378 in patients with cachexia. Overexpression of miR-378 catecholamine stimulated lipolysis in human adipocytes, suggesting that it may play a role in elevated lipolysis that results in adipose tissue loss in cancer cachexia (30).

\section{$\mathrm{miR}-30 \mathrm{~b} / \mathrm{c}$}

In addition to their function in regulation of adipogenesis (31), members of the miR-30 family have been shown to play a role in regulation of the thermogenic gene program in brown and beige fat (32). miR-30b is expressed in both human and mouse BAT (26). miR-30b and miR-30c expression is induced in BAT and subcutaneous WAT in response to cold exposure, $\beta 3$-adrenergic receptor activator CL-316,243, non-selective $\beta$-adrenergic receptor activator isoproterenol or the AMP inducer forskolin. Overexpression of miR-30b and miR-30c induced 
Ucp1 expression in BAT and subcutaneous WAT, both in vitro and in vivo. The positive effect of $\mathrm{miR}-30 \mathrm{~b} / \mathrm{c}$ on UCP1 expression seems to be mediated by targeting receptor-interacting protein 140 (Rip140). Rip140 is a transcriptional corepressor for nuclear receptors and is involved in the silencing of Ucp1 expression in white adipocytes through recruitment of chromatin remodeling enzymes, which enhance histone deacetylation and methylation of the Ucp1 enhancer and promoter (33).

\section{miR-455}

miR-455 was identified as a marker of BAT, acting downstream of BMP7 and cold-induced pathways to promote brown adipocyte lineage commitment (34). In both rodents and humans, miR455 is selectively expressed at higher levels in BAT compared with WAT, miR-455 overexpression promotes brown adipocyte differentiation in committed brown and white preadipocytes and in non-committed multipotent progenitor cells in vitro by inducing the expression of key regulators of adipogenesis such as Ppar $\gamma, \operatorname{Cebp} \alpha$, and $\operatorname{Cebp} \delta$, and important brown adipocyte markers Ucp1, Ppargc1 $\alpha$, Prdm16, and Cidea. Consistent with the in vitro findings, mice overexpressing miR-455 in adipose tissue using an aP2 promoter-driven transgene (FAT455 mice) exhibited an enhanced thermogenic capacity in response to cold or norepinephrine stimulation, which resulted from elevated Ucp1 expression in both BAT and subcutaneous WAT depots. As commonly seen in mouse models with higher energy expenditure, FAT455 mice showed an increase in food consumption. Under pair-fed condition, FAT455 mice were more resistant to weight gain and had improved insulin sensitivity and glucose tolerance compared to their wild-type littermates. miR-455 directly targets Runx1t1 and Necdin, both of which act as inhibitors of white and brown adipogenesis. In addition, miR-455 targets HIF1an. HIF1an is an asparaginyl hydroxylase enzyme regulating the activities of multiple cellular pathways through hydroxylation of Asn residues in substrates. In brown preadipocytes, HIF1an directly interacts with the AMPK $\alpha 1$ isoform and inhibits AMPK activity, at least in part, via asparaginyl hydroxylation. Therefore, miR-455 overexpression suppresses HIF1an expression, leading to removing an inhibitory signal of AMPK and allowing AMPK to exert its full function. Activated AMPK in turn triggers Ppargc1 $\alpha$ phosphorylation, which could eventually enhance its transcriptional activity and result in promotion of brown adipogenesis.

\section{miR-32}

miR-32 is a BAT-enriched miRNA, recently identified to be located in close proximity of a BAT specific super enhancer (35). miR-32 expression in BAT is induced by cold exposure, and it regulates the thermogenic gene program. Inhibition of miR-32 in mice using an antisense oligo (ASO) impaired the activation of thermogenic response upon cold challenge and reduced energy expenditure. The compromised thermoregulatory capacity was due to both lower UCP1 induction in BAT and reduced recruitment of beige cells in scWAT. Mice injected with miR-32 ASO failed to upregulate FGF21 expression in BAT in response to cold exposure. Thus, miR-32 inhibition resulted in decreased serum FGF21 levels, which led to reduced browning of scWAT upon cold exposure. Transducer of ErbB-2.1 (Tob1) was identified as a direct target of miR-32. Tob1 repression by miR-32 results in activation of p38 MAP kinase signaling and ATF2 transcription factor to elevate FGF21 expression in BAT. Secreted FGF21 from BAT communicates with scWAT to promote browning of this depot in response to prolonged cold challenge.

\section{miRNAs AS INHIBITORS OF BROWN ADIPOGENESIS}

\section{miR-27}

miR-27 negatively regulates white and brown adipogenesis in mice $(36-38)$ and humans $(39,40)$. Expression of miR-27a and miR-27b was downregulated in BAT and scWAT of mice in response to cold exposure. Inhibition of miR-27 in brown preadipocytes enhanced the expression of multiple key transcription factors including Ppar $\alpha$, Ppar $\gamma, \operatorname{Prdm} 16$, and Ppargc1 $\alpha$ and facilitated the differentiation toward Ucp1 expressing adipocytes. Conversely, miR-27 overexpression reduces the expression of Prdm16 and Ucp1 during brown and beige adipogenesis. In vitro luciferase assay results demonstrated that miR-27 directly targets the 3' UTR of both Prdm16 and Pparo, suggesting that the inhibitory effect of miR-27 on brown adipogenesis might be through modulation of these factors.

\section{miR-34a}

miR-34a was identified as a negative regulator of brown and beige formation in obese mice. Expression of miR-34a was positively associated with BMI in human (41) and is upregulated in DIO mice (42). Lentiviral-mediated knockdown of miR-34a in DIO mice promotes the browning of multiple white fat depots including perirenal WAT and gonadal WAT, as well as scWAT, and protects mice from the detrimental metabolic effects of high fat diet. Mechanistically, miR-34a targets FGFR1 and therefore attenuates FGF21 responsiveness and downstream signaling in adipose tissue. SIRT1 is another direct target of miR-34a that plays a critical role in transcriptional regulation of several brown and beige fat markers through deacetylation of Ppargc1a. Given that FGF21 signaling has also been linked to modulation of Ppargcla activity, miR-34a is likely to negatively regulate the expression of browning genes in obesity through suppression of Ppargcla transcriptional activity. Interestingly, the beneficial effects of miR-34a inhibition go beyond its browning effects, as it also improves FGF21 signaling in the liver, which contributes to improved overall metabolism and decreased adiposity.

\section{miR-133}

miRNA microarray analysis of BAT from mice exposed to cold for $24 \mathrm{~h}$ identified miR-133 as one the most downregulated miRNAs after cold exposure. miRNA-133 is highly enriched in cardiac and skeletal muscle lineages. It was shown that miRNA-133 negatively regulates brown adipogenesis through suppression of Prdm16. Inhibition of miRNA-133 in brown and beige preadipocytes increases Prdm16, Ppar $\gamma$, and Ppar $\alpha$ 
expression and results in the elevation of Ucp1 expression after differentiation. Downregulation of miR-133 by $\beta$-adrenergic stimulation was suggested to be mediated by the transcriptional factors from the Mef2 family, Mef2c, Mef2a, and Mef2d (28). miR-133a and miR-133b are also expressed in multipotent satellite cells in adult skeletal muscle and are involved in lineage commitment and fate decision between myogenic and brown adipose lineages, primarily by targeting Prdm16. Inhibition of miR-133 resulted in the formation of metabolically active brown adipocytes in regenerating tibialis anterior muscles, which in turn promotes energy expenditure and protects mice against DIO (43).

\section{miR-155}

miR-155 is highly expressed in preadipocytes isolated from the stromal-vascular fraction of BAT, and its expression is dramatically reduced in differentiated brown adipocytes (44). miR-155 targets adipogenic transcription factor $\operatorname{Cebp} \beta$, and therefore suppresses the development of adipogenic and thermogenic programs. $\operatorname{Cebp} \beta$ also directly binds to the distal site $\mathrm{E}$ in the miR-155 regulatory region, and negatively regulates miR-155. Therefore, miR-155 and Cebp $\beta$ form a double-negative feedback loop, which ensures efficient induction of $\operatorname{Cebp} \beta$ upon adipogenic induction. Overexpression of miR-155 in brown preadipocytes suppresses adipogenic differentiation and induction of the thermogenic program in brown adipocytes. Consistently, it was found that miR-155 is a downstream effector of TGF $\beta 1$ signaling, which is known to block adipogenesis in vitro (45) and in vivo (46) through modulating the transcriptional activity of C/EBPs (47). In vivo overexpression of miR-155 selectively impaired brown fat development, while WAT was not affected. Conversely, loss of miR-155 enhanced the thermogenic capacity of BAT and resulted in elevated levels of scWAT browning in cold (44).

\section{BAT AS AN ENDOCRINE ORGAN SECRETING MIRNAs}

In addition to the well-known function of BAT in non-shivering thermogenesis and energy expenditure, recent studies have established BAT as an endocrine organ that secretes several "batokine" (48-54) and "lipokine" $(55,56)$ molecules, which mediate the cross-talk between different tissues in the body. More recently, using the adipose tissue-specific Dicer KO mouse model (ADicer KO), it was shown that brown and WAT contribute a major fraction of circulating exosomal miRNAs. Importantly, elevated FGF21 expression observed in the liver of ADicer $\mathrm{KO}$ mice can be lowered by the injection of exosomes from the serum of wild-type donors. Adenoviral delivery of hsa-miR-302f to the BAT of mice resulted in suppression of a miR-302f $3^{\prime}$ UTR reporter in liver (57). These observations suggest that BAT-derived miRNAs modulate gene expression in the liver, and potentially in other organs, as well.

miR-99b level was strongly reduced in circulating exosomes of ADicer $\mathrm{KO}$ mice and was restored by BAT transplantation.

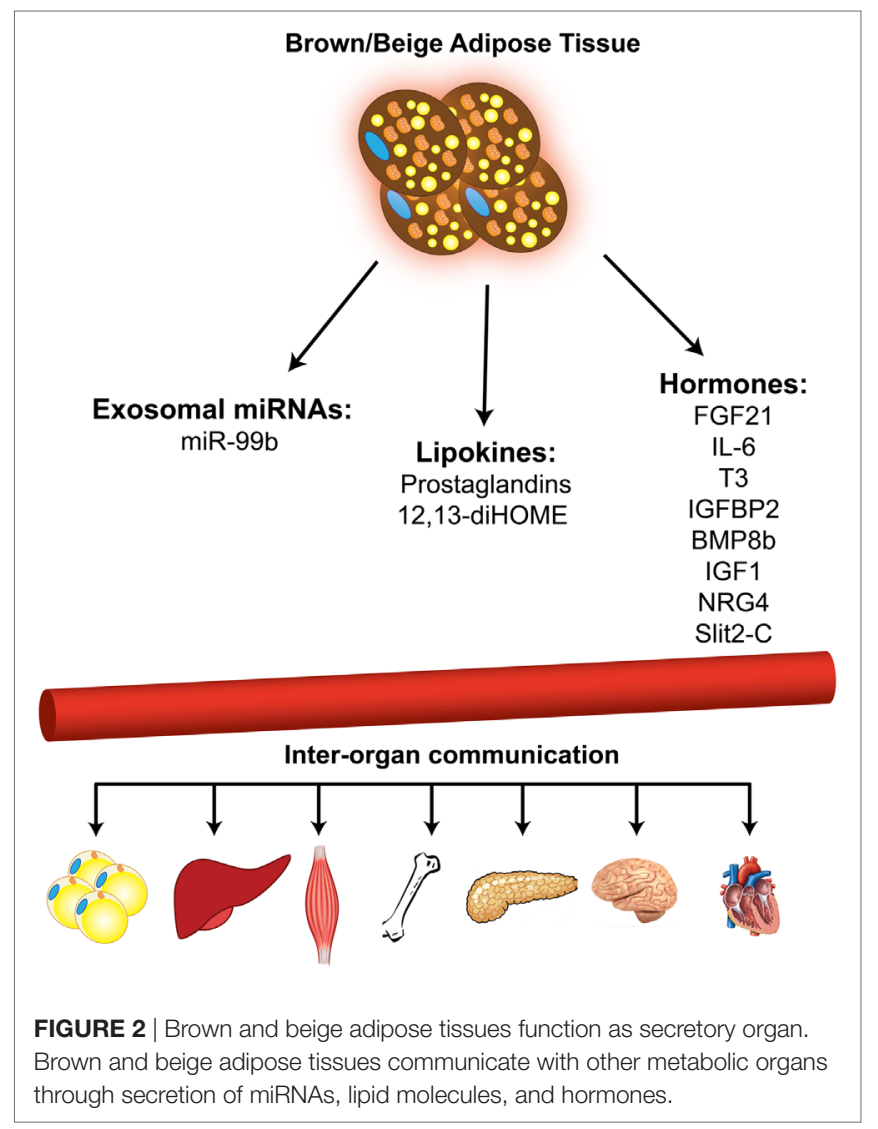

Treatment with exosomes from ADicer $\mathrm{KO}$ mice reconstituted with miR-99b dramatically reduced FGF21 expression in liver, indicating that adipose tissue-derived exosomal miR-99b regulates FGF21 expression in liver.

It has been shown that in vitro and in vivo activation of brown adipocytes results in increased release of exosomes (58). Exosomes released by cold-activated brown fat displayed a unique miRNA profile with higher levels of miR-34c* and lower levels of miR-92a compared with the brown fat of control mice maintained at room temperature. Interestingly, cold acclimation in humans lowered the exosomal miR-92a abundance in serum, and the level of miR-92a in human serum was shown to be inversely correlated with BAT activity. Based on this, exosomal miR-92a might serve as a biomarker for BAT activity in humans (Figure 2).

\section{CONCLUDING REMARKS}

Obesity is a major contributor to metabolic syndrome and disorders such as type 2 diabetes and cardiovascular diseases. Rediscovery of functional brown fat in adult humans has opened new avenues for utilizing its prominent capacity for fuel consumption and energy expenditure to combat obesity and its comorbidities (5-7). Investigation of therapeutic approaches focusing on BAT and its thermogenic capacity has gained considerable interest in the last several years. In addition to improving 
energy balance, activated BAT improves insulin sensitivity in both humans and mice $(59,60)$.

Due to their key roles in the regulation of gene expression networks, miRNAs are now considered a novel class of therapeutic targets. The recent trials for the application of miRNA mimics or inhibitors as drugs in humans have strengthened the idea of using miRNA-based therapeutics in humans for a range of diseases $(61,62)$. Some of the associated challenges that need to be overcome before establishing miRNA-based drugs include optimizing the tissue-specific delivery methods, characterizing and minimizing the off-target effects, and evaluating the toxicity and immunological responses. Therefore, we foresee that in the near future antagonizing or restoring specific miRNAs will be used as novel therapeutic strategies to target human BAT and WAT, and such therapies

\section{REFERENCES}

1. Hu E, Liang P, Spiegelman BM. AdipoQ is a novel adipose-specific gene dysregulated in obesity. J Biol Chem (1996) 271(18):10697-703. doi:10.1074/ jbc.271.18.10697

2. Montague CT, Farooqi IS, Whitehead JP, Soos MA, Rau H, Wareham NJ, et al. Congenital leptin deficiency is associated with severe early-onset obesity in humans. Nature (1997) 387(6636):903-8. doi:10.1038/43185

3. Steppan CM, Bailey ST, Bhat S, Brown EJ, Banerjee RR, Wright CM, et al. The hormone resistin links obesity to diabetes. Nature (2001) 409 (6818):307-12. doi:10.1038/35053000

4. Townsend KL, Tseng YH. Brown fat fuel utilization and thermogenesis. Trends Endocrinol Metab (2014) 25(4):168-77. doi:10.1016/j.tem.2013. 12.004

5. Cypess AM, Lehman S, Williams G, Tal I, Rodman D, Goldfine AB, et al. Identification and importance of brown adipose tissue in adult humans. N Engl J Med (2009) 360(15):1509-17. doi:10.1056/NEJMoa0810780

6. Nedergaard J, Bengtsson T, Cannon B. Unexpected evidence for active brown adipose tissue in adult humans. Am J Physiol Endocrinol Metab (2007) 293(2):E444-52. doi:10.1152/ajpendo.00691.2006

7. van Marken Lichtenbelt WD, Vanhommerig JW, Smulders NM, Drossaerts JM, Kemerink GJ, Bouvy ND, et al. Cold-activated brown adipose tissue in healthy men. N Engl J Med (2009) 360(15):1500-8. doi:10.1056/ NEJMoa0808718

8. Guerra C, Koza RA, Yamashita H, Walsh K, Kozak LP. Emergence of brown adipocytes in white fat in mice is under genetic control. Effects on body weight and adiposity. J Clin Invest (1998) 102(2):412-20. doi:10.1172/JCI3155

9. Wu J, Bostrom P, Sparks LM, Ye L, Choi JH, Giang AH, et al. Beige adipocytes are a distinct type of thermogenic fat cell in mouse and human. Cell (2012) 150(2):366-76. doi:10.1016/j.cell.2012.05.016

10. Lepper C, Fan CM. Inducible lineage tracing of Pax7-descendant cells reveals embryonic origin of adult satellite cells. Genesis (2010) 48(7):424-36. doi:10.1002/dvg.20630

11. Atit R, Sgaier SK, Mohamed OA, Taketo MM, Dufort D, Joyner AL, et al. Beta-catenin activation is necessary and sufficient to specify the dorsal dermal fate in the mouse. Dev Biol (2006) 296(1):164-76. doi:10.1016/j. ydbio.2006.04.449

12. Seale P, Bjork B, Yang W, Kajimura S, Chin S, Kuang S, et al. PRDM16 controls a brown fat/skeletal muscle switch. Nature (2008) 454(7207):961-7. doi:10.1038/nature07182

13. Farmer SR. Transcriptional control of adipocyte formation. Cell Metab (2006) 4(4):263-73. doi:10.1016/j.cmet.2006.07.001

14. Puigserver P, Wu Z, Park CW, Graves R, Wright M, Spiegelman BM. A coldinducible coactivator of nuclear receptors linked to adaptive thermogenesis. Cell (1998) 92(6):829-39. doi:10.1016/S0092-8674(00)81410-5

15. Seale P, Kajimura S, Yang W, Chin S, Rohas LM, Uldry M, et al. Transcriptional control of brown fat determination by PRDM16. Cell Metab (2007) 6(1): 38-54. doi:10.1016/j.cmet.2007.06.001 will benefit metabolism by improve energy expenditure and fuel homeostasis.

\section{AUTHOR CONTRIBUTIONS}

FS and Y-HT wrote the manuscript. HZ provided help and feedback.

\section{ACKNOWLEDGMENTS}

This work was supported in part by NIH grants R01 DK077097, R01 DK102898, and U24 DK076169-10S1 (to Y-HT) and P30 DK036836 (to Joslin Diabetes Center's Diabetes Research Center). The authors thank A. Slack and J. Darcy for editing the manuscript.

16. Cederberg A, Gronning LM, Ahren B, Tasken K, Carlsson P, Enerback S FOXC2 is a winged helix gene that counteracts obesity, hypertriglyceridemia, and diet-induced insulin resistance. Cell (2001) 106(5):563-73. doi:10.1016/ S0092-8674(01)00474-3

17. He L, Hannon GJ. MicroRNAs: small RNAs with a big role in gene regulation. Nat Rev Genet (2004) 5(7):522-31. doi:10.1038/nrg1379

18. Montano M. MicroRNAs: miRRORS of health and disease. Transl Res (2011) 157(4):157-62. doi:10.1016/j.trsl.2011.02.001

19. Ha M, Kim VN. Regulation of microRNA biogenesis. Nat Rev Mol Cell Biol (2014) 15(8):509-24. doi:10.1038/nrm3838

20. Mori MA, Raghavan P, Thomou T, Boucher J, Robida-Stubbs S, Macotela Y, et al. Role of microRNA processing in adipose tissue in stress defense and longevity. Cell Metab (2012) 16(3):336-47. doi:10.1016/j.cmet. 2012.07.017

21. Mori MA, Thomou T, Boucher J, Lee KY, Lallukka S, Kim JK, et al. Altered miRNA processing disrupts brown/white adipocyte determination and associates with lipodystrophy. J Clin Invest (2014) 124(8):3339-51. doi:10.1172/ JCI73468

22. Mudhasani R, Puri V, Hoover K, Czech MP, Imbalzano AN, Jones SN. Dicer is required for the formation of white but not brown adipose tissue. J Cell Physiol (2011) 226(5):1399-406. doi:10.1002/jcp.22475

23. Oliverio M, Schmidt E, Mauer J, Baitzel C, Hansmeier N, Khani S, et al. Dicer1-miR-328-Bace1 signalling controls brown adipose tissue differentiation and function. Nat Cell Biol (2016) 18(3):328-36. doi:10.1038/ncb3316

24. Johanson TM, Lew AM, Chong MM. MicroRNA-independent roles of the RNase III enzymes Drosha and Dicer. Open Biol (2013) 3(10):130144. doi:10.1098/rsob.130144

25. Macias S, Cordiner RA, Caceres JF. Cellular functions of the microprocessor Biochem Soc Trans (2013) 41(4):838-43. doi:10.1042/BST20130011

26. Guller I, McNaughton S, Crowley T, Gilsanz V, Kajimura S, Watt M, et al. Comparative analysis of microRNA expression in mouse and human brown adipose tissue. BMC Genomics (2015) 16:820. doi:10.1186/s12864015-2045-8

27. Sun L, Xie H, Mori MA, Alexander R, Yuan B, Hattangadi SM, et al. miR193b-365 is essential for brown fat differentiation. Nat Cell Biol (2011) 13(8):958-65. doi:10.1038/ncb2286

28. Trajkovski M, Ahmed K, Esau CC, Stoffel M. MyomiR-133 regulates brown fat differentiation through Prdm16. Nat Cell Biol (2012) 14(12):1330-5. doi:10.1038/ncb2612

29. Pan D, Mao C, Quattrochi B, Friedline RH, Zhu LJ, Jung DY, et al. MicroRNA-378 controls classical brown fat expansion to counteract obesity. Nat Commun (2014) 5:4725. doi:10.1038/ncomms5725

30. Kulyte A, Lorente-Cebrian S, Gao H, Mejhert N, Agustsson T, Arner P, et al. MicroRNA profiling links miR-378 to enhanced adipocyte lipolysis in human cancer cachexia. Am J Physiol Endocrinol Metab (2014) 306(3):E267-74. doi:10.1152/ajpendo.00249.2013

31. Karbiener M, Neuhold C, Opriessnig P, Prokesch A, Bogner-Strauss JG, Scheideler M. MicroRNA-30c promotes human adipocyte differentiation 
and co-represses PAI-1 and ALK2. RNA Biol (2011) 8(5):850-60. doi:10.4161/ rna.8.5.16153

32. Hu F, Wang M, Xiao T, Yin B, He L, Meng W, et al. miR-30 promotes thermogenesis and the development of beige fat by targeting RIP140. Diabetes (2015) 64(6):2056-68. doi:10.2337/db14-1117

33. Kiskinis E, Hallberg M, Christian M, Olofsson M, Dilworth SM, White R, et al. RIP140 directs histone and DNA methylation to silence Ucp1 expression in white adipocytes. EMBO J (2007) 26(23):4831-40. doi:10.1038/ sj.emboj.7601908

34. Zhang H, Guan M, Townsend KL, Huang TL, An D, Yan X, etal. MicroRNA-455 regulates brown adipogenesis via a novel HIFlan-AMPK-PGClalpha signaling network. EMBO Rep (2015) 16(10):1378-93. doi:10.15252/embr. 201540837

35. Ng R, Hussain NA, Zhang Q, Chang C, Li H, Fu Y, et al. miRNA-32 drives brown fat thermogenesis and trans-activates subcutaneous white fat browning in mice. Cell Rep (2017) 19(6):1229-46. doi:10.1016/j.celrep.2017.04.035

36. Lin Q, Gao Z, Alarcon RM, Ye J, Yun Z. A role of miR-27 in the regulation of adipogenesis. FEBS J (2009) 276(8):2348-58. doi:10.1111/j.1742-4658.2009. 06967.x

37. Sun L, Trajkovski M. miR-27 orchestrates the transcriptional regulation of brown adipogenesis. Metabolism (2014) 63(2):272-82. doi:10.1016/j. metabol.2013.10.004

38. Zhu Y, Zhang X, Ding X, Wang H, Chen X, Zhao H, et al. miR-27 inhibits adipocyte differentiation via suppressing CREB expression. Acta Biochim Biophys Sin (Shanghai) (2014) 46(7):590-6. doi:10.1093/abbs/gmu036

39. Chen SZ, Xu X, Ning LF, Jiang WY, Xing C, Tang QQ, et al. miR-27 impairs the adipogenic lineage commitment via targeting lysyl oxidase. Obesity (Silver Spring) (2015) 23(12):2445-53. doi:10.1002/oby.21319

40. Kang T, Lu W, Xu W, Anderson L, Bacanamwo M, Thompson W, et al. MicroRNA-27 (miR-27) targets prohibitin and impairs adipocyte differentiation and mitochondrial function in human adipose-derived stem cells. J Biol Chem (2013) 288(48):34394-402. doi:10.1074/jbc.M113.514372

41. Ortega FJ, Moreno-Navarrete JM, Pardo G, Sabater M, Hummel M, Ferrer A, et al. miRNA expression profile of human subcutaneous adipose and during adipocyte differentiation. PLoS One (2010) 5(2):e9022. doi:10.1371/ journal.pone.0009022

42. Fu T, Seok S, Choi S, Huang Z, Suino-Powell K, Xu HE, et al. MicroRNA 34a inhibits beige and brown fat formation in obesity in part by suppressing adipocyte fibroblast growth factor 21 signaling and SIRT1 function. Mol Cell Biol (2014) 34(22):4130-42. doi:10.1128/MCB.00596-14

43. Yin $\mathrm{H}$, Pasut A, Soleimani VD, Bentzinger CF, Antoun G, Thorn S, et al. MicroRNA-133 controls brown adipose determination in skeletal muscle satellite cells by targeting Prdm16. Cell Metab (2013) 17(2):210-24. doi:10.1016/j.cmet.2013.01.004

44. Chen Y, Siegel F, Kipschull S, Haas B, Frohlich H, Meister G, et al. miR-155 regulates differentiation of brown and beige adipocytes via a bistable circuit. Nat Commun (2013) 4:1769. doi:10.1038/ncomms2742

45. Ignotz RA, Massague J. Type beta transforming growth factor controls the adipogenic differentiation of 3T3 fibroblasts. Proc Natl Acad Sci U S A (1985) 82(24):8530-4. doi:10.1073/pnas.82.24.8530

46. Clouthier DE, Comerford SA, Hammer RE. Hepatic fibrosis, glomerulosclerosis, and a lipodystrophy-like syndrome in PEPCK-TGF-betal transgenic mice. J Clin Invest (1997) 100(11):2697-713. doi:10.1172/JCI119815

47. Choy L, Derynck R. Transforming growth factor-beta inhibits adipocyte differentiation by Smad3 interacting with CCAAT/enhancer-binding protein (C/EBP) and repressing C/EBP transactivation function. J Biol Chem (2003) 278(11):9609-19. doi:10.1074/jbc.M212259200

48. Chartoumpekis DV, Habeos IG, Ziros PG, Psyrogiannis AI, Kyriazopoulou VE, Papavassiliou AG. Brown adipose tissue responds to cold and adrenergic stimulation by induction of FGF21. Mol Med (2011) 17(7-8):736-40. doi:10.2119/molmed.2011.00075
49. Fernandez JA, Mampel T, Villarroya F, Iglesias R. Direct assessment of brown adipose tissue as a site of systemic tri-iodothyronine production in the rat. Biochem J (1987) 243(1):281-4. doi:10.1042/bj2430281

50. Hondares E, Iglesias R, Giralt A, Gonzalez FJ, Giralt M, Mampel T, et al. Thermogenic activation induces FGF21 expression and release in brown adipose tissue. J Biol Chem (2011) 286(15):12983-90. doi:10.1074/ jbc.M110.215889

51. Rosell M, Hondares E, Iwamoto S, Gonzalez FJ, Wabitsch M, Staels B, et al. Peroxisome proliferator-activated receptors-alpha and -gamma, and cAMP-mediated pathways, control retinol-binding protein-4 gene expression in brown adipose tissue. Endocrinology (2012) 153(3):1162-73. doi:10.1210/ en.2011-1367

52. Stanford KI, Middelbeek RJ, Townsend KL, An D, Nygaard EB, Hitchcox KM, et al. Brown adipose tissue regulates glucose homeostasis and insulin sensitivity. J Clin Invest (2013) 123(1):215-23. doi:10.1172/JCI62308

53. Whittle AJ, Carobbio S, Martins L, Slawik M, Hondares E, Vazquez MJ, et al. BMP8B increases brown adipose tissue thermogenesis through both central and peripheral actions. Cell (2012) 149(4):871-85. doi:10.1016/j. cell.2012.02.066

54. Yamashita H, Sato Y, Kizaki T, Oh S, Nagasawa J, Ohno H. Basic fibroblast growth factor (bFGF) contributes to the enlargement of brown adipose tissue during cold acclimation. Pflugers Arch (1994) 428(3-4):352-6. doi:10.1007/ BF00724518

55. Lynes MD, Leiria LO, Lundh M, Bartelt A, Shamsi F, Huang TL, et al. The cold-induced lipokine 12,13-diHOME promotes fatty acid transport into brown adipose tissue. Nat Med (2017) 23(5):631-7. doi:10.1038/nm.4297

56. Vegiopoulos A, Muller-Decker K, Strzoda D, Schmitt I, Chichelnitskiy E, Ostertag A, et al. Cyclooxygenase-2 controls energy homeostasis in mice by de novo recruitment of brown adipocytes. Science (2010) 328(5982): 1158-61. doi:10.1126/science.1186034

57. Thomou T, Mori MA, Dreyfuss JM, Konishi M, Sakaguchi M, Wolfrum C, et al. Adipose-derived circulating miRNAs regulate gene expression in other tissues. Nature (2017) 542(7642):450-5. doi:10.1038/nature21365

58. Chen Y, Buyel JJ, Hanssen MJ, Siegel F, Pan R, Naumann J, et al. Exosomal microRNA miR-92a concentration in serum reflects human brown fat activity. Nat Commun (2016) 7:11420. doi:10.1038/ncomms 11420

59. Chondronikola M, Volpi E, Borsheim E, Porter C, Annamalai P, Enerback S, et al. Brown adipose tissue improves whole-body glucose homeostasis and insulin sensitivity in humans. Diabetes (2014) 63(12):4089-99. doi:10.2337/db14-0746

60. Hanssen MJ, Hoeks J, Brans B, van der Lans AA, Schaart G, van den Driessche JJ, et al. Short-term cold acclimation improves insulin sensitivity in patients with type 2 diabetes mellitus. Nat Med (2015) 21(8):863-5. doi: $10.1038 / \mathrm{nm} .3891$

61. Bouchie A. First microRNA mimic enters clinic. Nat Biotechnol (2013) 31(7):577. doi:10.1038/nbt0713-577

62. Janssen HL, Reesink HW, Lawitz EJ, Zeuzem S, Rodriguez-Torres M, Patel K, et al. Treatment of HCV infection by targeting microRNA. N Engl J Med (2013) 368(18):1685-94. doi:10.1056/NEJMoa 1209026

Conflict of Interest Statement: The authors declare that the research was conducted in the absence of any commercial or financial relationships that could be construed as a potential conflict of interest.

Copyright (c) 2017 Shamsi, Zhang and Tseng. This is an open-access article distributed under the terms of the Creative Commons Attribution License (CC BY). The use, distribution or reproduction in other forums is permitted, provided the original author(s) or licensor are credited and that the original publication in this journal is cited, in accordance with accepted academic practice. No use, distribution or reproduction is permitted which does not comply with these terms. 